\title{
ALGUNOS RASGOS DE LA ECONOMIA ANDALUZA EN LA CRISIS. 1973-1985
}

\author{
Manuel DELGADO CABEZA*
}

\section{INTRODUCCION}

Andalucía llega a la antesala de la crisis con una economía desestructurada, con un cuerpo económico invertebrado que consta de dos partes que han ido progresivamente distanciándose: una moderna, apéndice y complemento de las economías centrales y otra autóctona, débil y en regresión, para la que cada vez se alejan más las posibilidades de integración en el sistema en condiciones de igualdad ${ }^{1}$.

Las tendencias «naturales» de la economía en este proceso de crecimiento divergente no sólo no fueron contrarrestadas por una política regional compensatoria, sino que se vieron en cierto modo alentadas por una estrategia que puso su acento en las políticas sectoriales, olvidando la dimensión espacial del crecimiento $^{2}$.

En lo que sigue se trata de presentar brevemente algunos de los rasgos que definen a la economía andaluza en el período $1973-1985^{3}$.

$\left({ }^{*}\right)$ Catedrático de Economía Aplicada. Universidad de Sevilla.

1. Entre estas dos partes existe una clara relación que invalida las tesis dualistas en su aplicación a la economía andaluza. Esta relación, así como el proceso que ha seguido la estructura económica de Andalucía hasta los alrededores de 1975 pueden verse con detalle en DELGADO CABEZA, M. Dependencia y marginación de la economía andaluza. Monte de Piedad y Caja de Ahorros de Córdoba, 1981.

2. Véase al respecto CUADRADO, J.R. «Economía y desequilibrios regionales en España» en $L a$ España de las Autonomías. Instituto de Estudios de Administración Local. Madrid, 1985.

3. Es necesario señalar aquí que la situación en que se encuentra la información estadística limita bastante las posibilidades de profundizar en el análisis; sobre todo en lo que se refiere al nivel de desagregación sectorial. Por otra parte se está produciendo un lamentable retraso en la aparición de algunos resultados como los del Padrón de Habitantes de 1986 que acentúan la urgencia de poner en marcha una organización estadística para cubrir las necesidades de información en Andalucía. 


\section{ESPECIALIZACION PRODUCTIVA}

En el cuadro I se tiene la composición relativa del empleo por sectores par. 1985 y los cambios experimentados en relación con la situación de 1973. E1 dicha tabla se observa, como rasgo más importante del período, la continuidaı del proceso de terciarización de la economía andaluza aproximadamente a mismo ritmo que en el período 1955-1973, pero a un ritmo superior a la medi: para España y al de Cataluña y el País Vasco, perteneciendo ya más de li mitad de la población empleada al sector de servicios. En la crịsis, la industria que venía incrementando su importancia, ha perdido peso relativo en la estruc tura económica de todas las áreas consideradas, en mayor intensidad en la áreas industrializadas. Sin embargo, se ha de tener en cuenta que nos estamo refiriendo a la evolución de una estructura porcentual, de modo que lo qu sucede en las regiones industrializadas es que la ganancia en peso del secto servicios no se hace a costa de la agricultura en la misma medida que en An dalucía. De aquí que no se pueda deducir de estas cifras que estas regione hayan soportado en mayor medida la crisis en el sector industrial.

CUADRO I

COMPOSICION DEL EMPLEO POR SECTORES (PORCENTAJES)

\begin{tabular}{|c|c|c|c|c|c|c|c|c|}
\hline & \multicolumn{4}{|c|}{1985} & \multicolumn{4}{|c|}{ Diferencias 1985-1973 } \\
\hline & 1 & 2 & 3 & 4 & 1 & 2 & 3 & 4 \\
\hline Andalucía $\ldots \ldots \ldots \ldots$ & 21,2 & 15,5 & 8,0 & 55,3 & $-12,3$ & $-2,4$ & $-1,7$ & 16,7 \\
\hline Cataluña …..................... & 6,1 & 34,0 & 6,6 & 53,2 & $-3,5$ & $-7,4$ & $-3,3$ & 14,2 \\
\hline País Vasco $\ldots . . . . . . . .$. & 5,9 & 39,5 & 6,7 & 47,7 & $-2,2$ & $-7,4$ & $-1,4$ & 10,9 \\
\hline España & 16,4 & 23,7 & 7,2 & 52,5 & $-8,4$ & $-2,9$ & $-2,4$ & 13,5 \\
\hline
\end{tabular}

1. Agricultura. 2. Industria. 3. Construcción. 4. Servicios.

Para perfilar mejor los rasgos de la estructura productiva andaluza en cuan to a su especialización dentro de la economía española hemos jerarquizad، los sectores productivos teniendo en cuenta la participación de cada uno di ellos en su correspondiente total para España (cuadro II), apareciendo en di cho cuadro una clasificación análoga para Cataluña como prototipo de áre: industrializada. Como se ve Andalucía continúa en las antípodas de las espe cialización regional con respecto a Cataluña.

Para la economía andaluza, pueden distinguirse tres grandes bloques, a lo que nos referimos a continuación. 


\section{CUADRO II}

PARTICIPACION DE LOS DISTINTOS SECTORES EN EL CORRESPONDIENTE TOTAL DE ESPAÑA (\% PRODUCCION)

\begin{tabular}{|c|c|c|c|}
\hline Andalucía & 1985 & Cataluña & 1985 \\
\hline Agricultura $\ldots$ & 26,8 & Químicas . ........................ & 36,8 \\
\hline Pesca & 21,9 & Textil, Calzado y Confección & 36,8 \\
\hline Alimentarias $\quad \ldots \ldots \ldots \ldots \ldots \ldots \ldots \ldots \ldots \ldots \ldots \ldots \ldots \ldots$ & 15,9 & Caucho, Plastico y O.M. ............. & 32,1 \\
\hline Servicios Públicos ..................... & 15,6 & 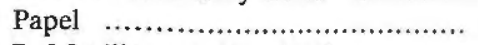 & 31,3 \\
\hline Servicio Doméstico ..................... & 15,1 & P. Metálicos y Maquinaria ............ & 25,3 \\
\hline Edif. y Obras Publicas $\quad . . . . . . . . . . . .$. & 15,1 & Enseñanza y Sanidad .................. & 22,7 \\
\hline Minerales y Metales . .................. & 13,6 & Rec. y Reparaciones ................... & 21,8 \\
\hline 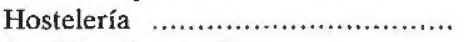 & 13,3 & 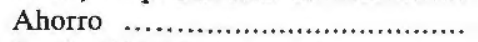 & 20,9 \\
\hline Servicios Comerciales $\quad . . . \ldots \ldots \ldots \ldots \ldots$ & 12,4 & Producción Energía y Agua .......... & 20,8 \\
\hline Prop. de Viviendas .................... & 12,2 & 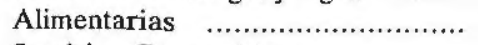 & 20,7 \\
\hline Otros Servicios & 12,2 & Servicios Comerciales ................. & 20,9 \\
\hline Rec. y Reparaciones .................. & 11,9 & Prop. de Viviendas ..................... & 19,7 \\
\hline Transportes y Comunicaciones $\quad . . .$. & 11,3 & Otros Servicios & 19,1 \\
\hline Minerales no Metálicos . ............... & 10,8 & Minerales no Metálicos ................ & 19,0 \\
\hline Químicas ................................... & 10,6 & Madera y Corcho ........................ & 18,7 \\
\hline Enseñanza y Sanidad $\quad . . \ldots \ldots \ldots \ldots \ldots \ldots$ & 10,6 & Servicio Domestico .................... & 17,8 \\
\hline 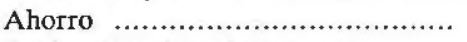 & 8,6 & Transportes y Comunicaciones ..... & 17,3 \\
\hline Producción Energía y Agua ......... & 8,2 & 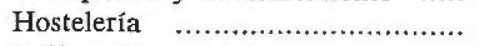 & 16,8 \\
\hline Madera y Corcho ....................... & 6,8 & Edif. y Obras Públicas ................ & 14,7 \\
\hline Textil, Calzado y Confección ....... & 5,9 & 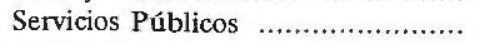 & 14,0 \\
\hline 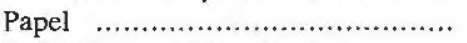 & 5,7 & 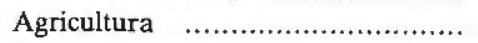 & 7,4 \\
\hline P. Metálicos y Maquinaria ........... & 5,5 & 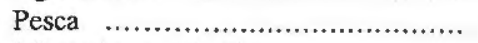 & 6,6 \\
\hline Caucho, Plástico y O.M. ............ & 4,5 & Minerales y Metales .................... & 4,1 \\
\hline
\end{tabular}

Fuente: Elaboración propia a partir de Renta Nacional de España. Banco de Bilbao.

\section{AGRICULTURA E INDUSTRIAS ALIMENTARIAS}

Las actividades primarias ocupan en 1985 los primeros lugares, con la particularidad de que la agricultura ha experimentado un avance importante en cuanto a su cuota de participación en la producción española durante la crisis ( $20,5 \%$ en $1973,26,8 \%$ en 1985), que nos anuncia una profundización en la especialización agraria, ahora como se verá, en peores condiciones para la economía andaluza que en otras épocas. Recuérdese en este sentido que a mediados de los años cincuenta Andalucía transformaba en gran medida su producción agraria aportando el $25 \%$ de la producción agroalimentaria española.

En 1985 la decadencia en la que el sector agroalimentario se ha visto sumido desde entonces lo lleva a contribuir con sólo un 15,9 \%. En Cataluña ha sucedido justo lo contrario. En tres décadas Cataluña ha doblado su aportación a la producción alimentaria española. 
A este respecto es necesario subrayar que no sólo ha continuado el declivı de las alimentarias andaluzas sino que durante la crisis se ha acentuado el rit mo de su regresión ( $-1,1 \%$ anual acumulativo frente a $-0,5 \%$ en 1964-73) ${ }^{4}$ Este deterioro relativo del aparato transformador de los productos agrario contrasta con la necesidad que se sostiene desde muchos puntos de vista d, contar con un complejo agroalimentario andaluz y significa desaprovechar un: de las mayores, por no decir la mayor potencialidad de la economía andaluza

En Andalucía este incremento de la especialización agraria acompañado d، un retroceso de las industrias agroalimentarias supone que se ha debilitad، uno de los escasos eslabones intersectoriales de la economía andaluza. Se hi profundizado la desarticulación del tejido económico andaluz en un sector lí der por su dinamicidad en términos de inversión en los años 80 en España ${ }^{5}$

La reconstrucción ahora de este sector autóctono, en gran parte desmante lado en los treinta años que aquí se han considerado, podría dar lugar a l: implantación de una industria llegada desde fuera en un sector claramente do minado por las multinacionales, resultando un sector agroalimentario cualita tivamente muy distinto y alejado de un esquema de industrialización endó gena.

\section{LOS SERVICIOS}

En segundo lugar, como en 1973, se puede distinguir un grupo de sectore de servicios cuyos porcentajes de participación no difieren mucho de los di 1973. En efecto, en su conjunto el sector servicios en 1973 aportaba el 12,t \% del valor añadido por este sector en España y en 1985 este porcentaje e de un $12,5 \%$. Su situación, con respecto al inicio de la crisis, no ha variad, en cuanto al grado de participación en el total español, aunque el terciario e un sector en el que se están produciendo profundos cambios que no es posibl detectar al nivel de agregación de las estadísticas en la actualidad y cuyo aná lisis para Andalucía exigiría una investigación con este propósito ${ }^{6}$.

4. En relación con este hecho en Andalucía se ha perdido casi la mitad del empleo en las industria alimentarias entre 1973 y 1985 (el 46,3 \% según la Renta nacional de España del Banco de Bilbao:

5. AURIOLES, J.: «Dinamicidad industrial española en los años 80 ». Papeles de Economía, n. ${ }^{\circ}{ }^{3}$ ' 1988.

6. Existen algunos trabajos en los que se ponen de relieve estos cambios para España. Entre ellc cabe citar los de CUADRADO ROURA, J.R. «La evolución del empleo en los servicios» Papele de Economia, $\mathrm{n} .{ }^{\circ} 26,1986$, del RIO GOMEZ, C. "Los servicios a las empresas: una explicació del crecimiento del sector terciario», Economistas, n. ${ }^{\circ} 29,1988$ y en el mismo número ALCAIDE C. «El proceso de terciarización de la economía española». 
En los servicios andaluces se ha mantenido la productividad muy por debajo de la del centro. Aproximadamente el valor añadido por empleo continúa siendo un veinticinco por ciento menor en Andalucía que en Cataluña. Se ha producido una penetración importante de las formas productivas modernas, como lo demuestra, por una parte el aumento de la proporción de asalariados del sector que ahora se sitúa al mismo nivel que en Cataluña (74 \%) y por otra la disminución del número de empresarios. A su vez el coeficiente de variación para la productividad de los subsectores dentro de los servicios (cuadro 3) ha pasado de 25,9 en 1975 a 30,0 en 1985. Todo ello apunta en la dirección de un incremento en la distancia entre los servicios «modernos» y la parte del sector que comprende un conjunto de actividades de muy bajo valor añadido por persona empleada.

\section{CUADRO III}

EL SECTOR SERVICIOS EN 1985

\begin{tabular}{|c|c|c|c|c|}
\hline \multirow[b]{2}{*}{ Sectores } & \multicolumn{2}{|c|}{ Andalucía } & \multicolumn{2}{|c|}{ Cataluña } \\
\hline & $\begin{array}{c}\text { Empleo } \\
\text { (miles) }\end{array}$ & $\begin{array}{l}\text { V.A.B.* } \\
\text { Empleo }\end{array}$ & $\begin{array}{c}\text { Empleo } \\
\text { (miles) }\end{array}$ & $\begin{array}{l}\text { V.A.B.* } \\
\text { Empleo }\end{array}$ \\
\hline 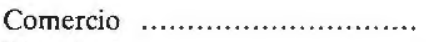 & 214,5 & $1.942,6$ & 307,6 & $2.235,3$ \\
\hline Hostelería $\quad$........................... & 93,3 & $2.225,5$ & 99,1 & $2.645,9$ \\
\hline Transportes .......................... & 80,8 & $2.911,3$ & 107,8 & $3.339,9$ \\
\hline Creditos y Seguros $\quad \ldots \ldots \ldots \ldots \ldots \ldots$ & 38,1 & $4.342,1$ & 69,6 & $5.774,1$ \\
\hline Enseñanza y Sanidad $\ldots . . \ldots \ldots \ldots$ & 24,8 & $2.617,2$ & 44,7 & $3.110,4$ \\
\hline Servicios Públicos $\ldots \ldots \ldots \ldots \ldots \ldots \ldots$ & 24,6 & $2.034,7$ & 223,7 & $2.135,5$ \\
\hline Servicios Diversos $\quad \ldots \ldots \ldots \ldots \ldots \ldots$ & 174,8 & $2.140,8$ & 211,1 & $2.699,4$ \\
\hline 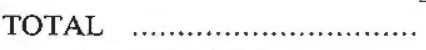 & 650,9 & $2.417,3$ & $1.063,6$ & $2.981,3$ \\
\hline
\end{tabular}

(*) En miles de ptas.

Fuente: Elaboración propia a partir de Renta Nacional de España. Banco de Bilbao.

Comercio, Hostelería y Servicios diversos son los subsectores donde la productividad es menor.

La Hostelería comprende actividades de naturaleza muy dispar, incluyendo tanto a los modernos establecimientos hoteleros como bares de todo tipo, cafeterías y restaurantes. La cifra tan baja que representa el valor añadido por persona y el alto porcentaje de empleos que absorbe $(14,3 \%)$ son indicios de la proliferación de pequeños negocios familiares. El padrón de habitantes de 1975 daba una cifra de trabajadores autóctonos más ayuda familiar en Hostelería que suponía el 15,1 \% del total de activos, porcentaje muy superior 
al de la media andaluza para los servicios (10,8\%). En 1981 trabajadores at tónomos más ayuda familiar pasan a representar prácticamente la cuarta part de los activos del sector $(24,5 \%)$, siendo la media para Andalucía en los se vicios de un $15 \%$.

Se ha incrementado, pues, el peso de las actividades «marginales» en Hos telería en mayor medida de lo que lo ha hecho en los servicios en general

El Comerio, tanto por su valor añadido como desde el punto de vista de empleo sigue siendo la actividad más importante de los servicios.

La evolución del empleo en el comercio (cuadro 4) nos muestra una mayo expansión de este subsector en Andalucía en la década 1960-70 y una contrac ción relativa del sector en 1970-81, mientras en Cataluña y en otras regione centrales el empleo en la actividad comercial ha crecido más deprisa en est última década ${ }^{7}$.

\section{CUADRO IV}

EVOLUCION DEL EMPLEO EN EL COMERCIO

(Miles)

\begin{tabular}{|c|c|c|c|}
\hline & & Andalucía & Cataluña \\
\hline 1950 & 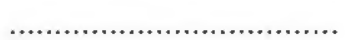 & 115,2 & 116,8 \\
\hline 1960 & 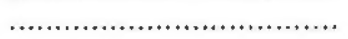 & 138,8 & 178,3 \\
\hline 1970 & (.) & 174,2 & 201,1 \\
\hline 1981 & 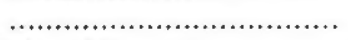 & 205,4 & 264,5 \\
\hline
\end{tabular}

Fuente: Censos de población (I.N.E.).

Lo ocurrido en el último período se puede apreciar mejor con ayuda de cuadro 5 donde se tiene la distribución de la población ocupada según su situa ción profesional. En dicha tabla puede observarse que a la vez que se conserv el número de empresarios y aumenta en términos absolutos el número de asa lariados hay un incremento del grupo de autónomos y ayuda familiar, que pa san de suponer en 1970 un $18 \%$ a un $31 \%$ en 1980 . Si atendemos a los dato que nos proporciona otra fuente estadística para el período 1973-1985, ha una cierta concordancia con lo que se acaba de comentar. La tabla 6 nos mues tra, junto a un aumento del peso de los no asalariados en Andalucía, una re ducción en las diferencias de productividad frente a Cataluña.

7. Este fenómeno se ve confirmado por las cifras de empleo para el comercio en 1973 y 1985 c Renta Nacional de España del Banco de Bilbao. 


\section{CUADRO V}

OCUPACION SEGUN SU SITUACION PROFESIONAL

(Miles)

\begin{tabular}{|c|c|c|}
\hline Situación profesional & 1970 & 1981 \\
\hline 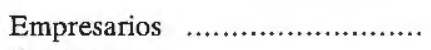 & 17,1 & 17,1 \\
\hline 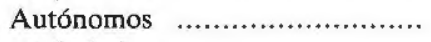 & 47,1 & 55,2 \\
\hline Asalariados ............................ & 100,5 & 115,7 \\
\hline Ayuda familiar $\quad \ldots \ldots \ldots \ldots \ldots \ldots \ldots$ & 7,6 & 12,8 \\
\hline 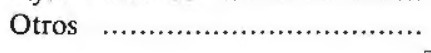 & 1,9 & 4,6 \\
\hline 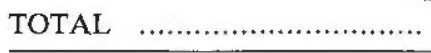 & 174,2 & 205,4 \\
\hline
\end{tabular}

Fuente: Censos de Población (I.N.E.).

\section{CUADRO VI}

EMPLEO Y VALOR AÑADIDO POR PERSONA

\begin{tabular}{llll}
\hline Comunidad & Asalariados $\left({ }^{*}\right)$ & No asalariados $(*)$ & V.A.B./Empleo $\left({ }^{* *}\right)$ \\
\hline
\end{tabular}

1973

\begin{tabular}{|c|c|}
\hline Andalucía $\quad \ldots \ldots \ldots \ldots \ldots \ldots$ & 103,0 \\
\hline (.). & 141,6 \\
\hline
\end{tabular}

1985

\begin{tabular}{|c|c|c|}
\hline Andalucía $\quad \ldots . . . \ldots \ldots \ldots \ldots$ & 97,6 & 116,8 \\
\hline Cataluña $\quad . . \ldots \ldots \ldots \ldots \ldots \ldots . . . . . . .$. & 163,2 & 144,4 \\
\hline
\end{tabular}

(*) Miles de empleos.

(**) Miles de pesetas.

Fuente: Renta Nacional de España. Banco de Bilbao.

Estas cifras reflejan un incremento del tamaño medio de los establecimientos comerciales que responden a formas productivas modernas, al tiempo que se produce un aumento de la importancia de las formas precapitalistas de comercio (pequeños comerciantes autónomos). No tiene lugar ahora, en su conjunto, y como balance, destrucción de estas formas productivas "atrasadas" como ocurría en 1964-1973; la crisis conlleva la pervivencia e incluso la expansión de estas actividades de fácil acceso y baja productividad. En este sentido puede decirse que se ha producido, también en este ámbito de la realidad económica de Andalucía, una mayor separación entre lo nuevo y lo marginal, que va acogiendo a un sector cada vez más amplio de la población ocupada. 


\section{LA INDUSTRIA}

El tercer grupo que se identifca en el cuadro 2 es el de las actividades indus triales que ocupan los últimos lugares, con porcentajes que han permanecids aproximadamente al mismo nivel que en 1973. Para aproximarnos a alguno: rasgos de la industria andaluza en 1985 consideremos en primer lugar lá composición del empleo y una medida de la productividad global del secto: (cuadro 7).

CUADRO VII

LA INDUSTRIA. 1985. PORCENTAJE DE NO ASALARIADOS

Y VALOR AÑADIDO POR PERSONA

\begin{tabular}{|c|c|c|}
\hline & N. ${ }^{\circ}$ de asalariados/Empleo & V.A.B./Empleo \\
\hline Andalucía $\quad . . . . . . . \ldots \ldots \ldots \ldots \ldots . . . . . . . .$. & 13,8 & 2.522 \\
\hline Cataluña $\ldots . . . \ldots \ldots \ldots \ldots \ldots \ldots \ldots \ldots . . . . . . . . . .$. & 9,2 & 2.688 \\
\hline
\end{tabular}

Fuente: Renta Nacional de España. Banco de Bilbao.

Como se ve, en 1985 continua siendo mayor en Andalucía la pervivenci: de formas tradicionales de producción. Los porcentajes de no asalariados e1 la industria andaluza eran en 1973 de $15,0 \%$, luego puede decirse que en l: crisis ha disminuido, aunque muy ligeramente, el peso del sector tradiciona en la industria andaluza, en contra de lo que sucedió en el período 1955-7. en el que se redujo a la mitad la importancia relativa de los no asalariado de la industria. Se ha ralentizado ahora la caida relativa de las formas produc tivas tradicionales en el sector industrial. Sin embargo este hecho está condi cionado por la evolución del empleo industrial en términos absolutos. En efec to, la evolución fuertemente decreciente del empleo industrial en el períod، 73-85 a la que nos referiremos más adelante hace que aparezca como más levi esta disminución de los no asalariados, porque desde el punto de vista del nú mero de empleos se pierde el 34,7\% del total de no asalariados existentes e1 1973, es decir, el ritmo es aproximadamente el mismo que en 1964-73. 


\section{LA DINAMICA SECTORIAL}

Si ahora consideramos la dinámica de la especialización productiva y seleccionamos los sectores que aumentan o disminuyen significativamente su participación en la producción española equivalente (cuadro 8) observamos que, en consonancia con lo que se decía anteriormente, la agricultura es el único sector dinámico en la crisis, mientras que aparecen como sectores en regresión actividades industriales como la Minería, Madera y corcho, Cerámica, vidrio y cemento, Industrias metálicas e Industrias agroalimentarias.

\section{CUADRO VIII}

DINAMICA DE LA ESPECIALIZACION SECTORIAL EN ANDALUCIA 1973-1985

\begin{tabular}{|c|c|c|c|}
\hline Agricultura & 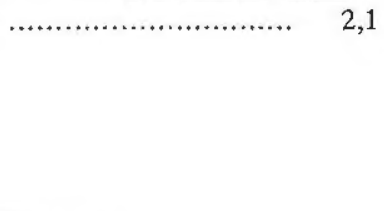 & 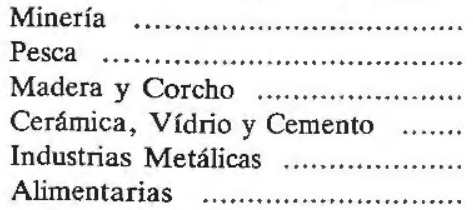 & $\begin{array}{l}-4,4 \\
-3,5 \\
-3,0 \\
-1,7 \\
-1,6 \\
-1,1\end{array}$ \\
\hline
\end{tabular}

Fuente: Elaboración propia.

Se puede decir, por tanto, que durante la crisis se ha frenado el proceso de traslación de ciertas producciones industriales a Andalucía, a pesar de que, como se ha indicado en algunos trabajos, en España «se ha detenido e incluso disminuye la concentración espacial de las actividades industriales», al menos hasta $1983^{8}$.

Por otra parte ha tenido lugar una clara profundización en la especialización tradicional de la economía andaluza continuando a su vez el proceso de deterioro relativo de algunas industrias que tenían cierto peso en la región (Alimentarias, textil, madera y corcho, cerámica vidrio y cemento). Estos sectores se corresponden con actividades en las que las unidades de producción poseen en general dimensiones reducidas que exigen una inversión media menor y hacia las que se venía orientando el capital autóctono ante condiciones de acceso

8. CUADRADO, J.R.: «Cambios en la distribución espacial de la industria en España» en Homenaje al profesor G. Barbancho. Consejería de Hacienda. Junta de Andalucía. En prensa. A partir de 1983, ante una nueva etapa de mayor aceleración del crecimiento se regresa a los niveles de concentración industrial anteriores a la crisis de los setenta. De cualquier modo, continua siendo la industria química la que mayor volumen de inversiones absorbe en valor absoluto. Véase GIRALDEZ, E. "La inversión industrial, algunas consideraciones en torno a su comportanuiento sectorial y espacial durante la crisis». SITUACION, n, ${ }^{\circ} 1,1986$. 
más favorables ${ }^{9}$. Se trata de sectores donde la pequeña y mediana empresa ha podido desplegar mejor su capacidad de defensa y adaptación ante la crisis. La aportación decreciente de Andalucía a la producción española en este tipc de actividades pone de relieve las deficiencias de la estructura empresarial an. daluza en este ámbito de la pequeña y mediana empresa ${ }^{10}$ y supone una pérdida de posiciones para una estructura empresrial autóctona que ve debilitarse aún más si cabe su ya precaria situación.

El progresivo empobrecimiento industrial relativo queda ratificado por los análisis sobre localización espacial de las inversiones industriales, en los que Andalucía aparece como una zona que desde 1974 hasta 1980 empeora su situación con respecto a períodos anteriores ${ }^{11}$. En especial desde los años $8 \mathrm{C}$ Andalucía, desde el punto de vista de la inversión industrial se configura como un área en decadencia ${ }^{12}$ en la que sólo Cádiz figura como de alta dinamicidad por inversiones muy puntuales de elevada dimensión ${ }^{13}$ y Sevilla se encuentra «en estancamiento». El resto conforma un espacio en declive incluido en una cuarta y última categoría «no solamente por cuanto tiene un peso actual poco significativo en el conjunto del sistema industrial nacional, sino también porque su participación en el movimiento de nuevas industrias es relativamente reducida. Es de prever la ampliación futura de los diferenciales de aportación al PIB industrial entre estas zonas y las incluidas en las otras tres categorías» (1. Alta, 2. Baja, 3. En estancamiento, 4. En decadencia $)^{14}$.

No parece extraño que la crisis haya afectado en mayor medida a una estructura industrial tal vulnerable como la que nos encontramos al inicio del período depresivo, máxime cuando esta etapa histórica «ha sido testigo de una clara pérdida de peso de la política regional dentro de la política económica, quedando el panorama dominado prácticamente por los impulsos del propio sistema económico y por las políticas de carácter nacional y sectorial» ${ }^{15}$.

9. DELGADO CABEZA, M.: Dependencia y marginación de la economía andaluza. Op. cit., pág. 152.

10. Véase GUZMAN, J.: La financiación de las PYMES en Andalucía. IDR, Sevilla 1986.

11. Véase el trabajo de GIRALDEZ, E. y VILLEGAS, P. «El componente espacial de la inversión industrial durante el período 1969-80». SITUACION 1984/3, pág. 17.

12. AURIOLES, J.: «Dinamicidad industrial española en los años 80». PAPELES DE ECONOMIA, n. ${ }^{\circ} 34,1988$,

13. Concretamente es la inversión de la General Motors la que explica la situación de Cádiz.

14. AURIOLES, J. Art. cit., pág. 394.

15. CUADRADO, J.R.: «Tendencias económico-regionales antes y después de la crisis en España». PAPELES DE ECONOMIA, ฉ. ${ }^{\circ} 34$. 1988, pág. 32. 


\section{CONSIDERACIONES FINALES}

En primer lugar es necesario advertir que en esta primera aproximación a lo acontecido en la economía andaluza en el período 1973-1985 y con el niveI de desagregación de la información estadística con que se cuenta, no se ha pretendido ir más allá de señalar algunos rasgos en lo que se refiere al comportamiento sectorial de la economía andaluza en la etapa antes citada. Es preciso una mayor profundización, así como la consideración de otras variables y otros aspectos para poder armar una interpretación consistente e integradora de la evolución de la estructura económica y social de Andalucía en la crisis.

No obstante aquí se han podido detectar ciertas tendencias que se pueden resumir como sigue:

1. Se advierte una profundización en la especialización productiva agraria, pasando la agricultura andaluza de aportar el 20,5\% a la producción española equivalente en 1973 a un $26,8 \%$ en 1985 . En este sentido aparece la agricultura como único sector dinámico de la crisis.

2. Por otra parte este auge de la agricultura coincide con el declive del sector agroalimentario, que continua su regresión, aunque ahora a mayor ritmo que en períodos anteriores. No sólo su disminución en cuanto a participación en las agroalimentarias españolas es importante $(19,5 \%$ en $1973,15,9$ en 1985), sino que en estos años se produce una pérdida de casi la mitad del empleo existente en $1973(43,3 \%)$.

Las posibilidades potenciales se ven, pues, disminuidas ante este distanciamiento entre una agricultura creciente y un sector agroalimentario en retroceso, al tiempo que se debilita uno de los escasos eslabones intersectoriales en la economía andaluza, profundizándose así la desarticulación del tejido productivo en Andalucía.

En este contexto, si como estrategia sectorial en Andalucía se apunta hacia el desarrollo del complejo agroalimentario, como se hace en el Programa Andaluz de Desarrollo Económico 1987-1990, cuyo núcleo gira alrededor de la industria agroalimentaria, es difícil pensar en la reconstrucción de este sector autóctono, débil y en buena parte desmantelado, bajo el impulso de la «libre» competencia, cuando de lo que se trata en buena medida es de desbancar del mercado a las grandes corporaciones que han resultado de un largo proceso de concentración del capital industrial en el centro; el resultado de esta pretensión puede ser, como se está evidenciando ya en algunos casos, un sector agroalimentario cualitativamente muy distinto y alejado de un esquema de industrialización endógena.

3. En la estructura económica andaluza se ha debilitado el peso del sector industrial, en el que se detecta una disminución de las formas productivas tradicionales a un ritmo aproximadamente igual al que venía aconteciendo para 
1964-1973. Al mismo tiempo, no aparecen entre los sectores más dinámico actividades industriales, de tal modo que se ha frenado el proceso de traslació, de algunas actividades transformadoras hacia Andalucía, aunque en valor ab soluto ha sido la industria Química, que como se sabe funciona como un: actividad de enclave en Andalucía, la que mayor volumen de inversiones ab sorbió.

4. Ha continuado el deterioro relativo de actividades como alimentarias textil, madera y corcho o cerámica, vidrio y cemento, que se correspondes con aquellas hacia las que se había venido orientando el empresariado autóc tono ante condiciones de acceso más favorables. La aportación decreciente di Andalucía en este tipo de actividades pone de relieve las dificultades por la que atraviesa este empresariado y supone una pérdida relativa de posicione para una estructura empresarial que ve empeorar su ya precaria situación

5. El sector servicios viene ganando peso relativo a mayor ritmo de lo qu viene sucediendo en otras áreas. Continua, por tanto el proceso de terciariza ción de la economía andaluza.

Como ya se había mostrado para períodos anteriores, el conjunto de activi dades de servicios en Andalucía no ha venido funcionando en sincronía col el resto del cuerpo económico en el sentido que preconizan los modelos con vencionales. En consecuencia, un crecimiento de la importancia de los servi cios, en paralelo a un retroceso como el que se acaba de reseñar del aparatı productivo industrial no puede ser interpretado como elemento positivo, dina mizador de la estructura económica de Andalucía. Al contrario, más bien con duce a pensar que el sector servicios continua su andadura como residuo el el proceso de subdesarrollo, siendo su crecimiento un síntoma claro de la in capacidad en el resto del sistema productivo para dar cabida a actividades empleo que han de refugiarse en este sector.

6. En este contexto, la productividad se mantiene muy baja en 1973-1985 incrementándose la heterogeneidad intrasectorial; es decir, que ahora las dife rencias entre productividades correspondientes a distintas actividades de lo servicios es mayor que en el período 1964-1973. Ello significa un increment en la distancia entre los servicios «modernos» y el conjunto de actividades d menor productividad o marginales.

El peso de estas actividades marginales se ha incrementado sobre todo et Hostelería, donde trabajadores autónomos mas ayuda familiar pasan de repre sentar un $15,1 \%$ en 1975 a un $24,5 \%$ del empleo en 1985. También en e comercio, la actividad más importante del terciario en Andalucía, se ha pro ducido una mayor separación entre lo «moderno» y lo «marginal», ámbito est que acoge a una parte creciente de la población ocupada. 